\title{
Anabases
}

ANABASES Traditions et réceptions de l'Antiquité

$2 \mid 2005$

Varia

\section{Mikhail I. Rostovtzeff et Max Weber : une rencontre manquée de l'histoire avec l'économie}

Hinnerk Bruhns

\section{(2) OpenEdition}

\section{Journals}

Édition électronique

URL : http://journals.openedition.org/anabases/1587

DOI : 10.4000/anabases. 1587

ISSN : 2256-9421

Éditeur

E.R.A.S.M.E.

Édition imprimée

Date de publication : 1 octobre 2005

Pagination : 79-99

ISSN : 1774-4296

\section{Référence électronique}

Hinnerk Bruhns, « Mikhail I. Rostovtzeff et Max Weber : une rencontre manquée de l'histoire avec

l'économie », Anabases [En ligne], 2 | 2005, mis en ligne le 01 juillet 2011, consulté le 21 octobre 2019. URL : http://journals.openedition.org/anabases/1587 ; DOI : 10.4000/anabases.1587 
Anabases 2 (2005), p. 79-99

\section{Mikhail I. Rostovtzeff et Max Weber : une rencontre manquée de l'histoire avec l'économie *}

HINNERK BRUHNS

\section{Weber et Rostovtzeff : un problème inexistant pour l'historiographie relative à l'économie antique}

Si les itinéraires sCientifiques de Max Weber (1864-1920) et de Mikhail I. Rostovtzeff (1870-1952) se sont croisés, dans la première décennie du XXe siècle, dans les faits, les deux hommes ne se sont apparemment jamais rencontrés personnellement. Cela eût pourtant été tout à fait possible à la faveur d'un des séjours de Rostovtzeff en Allemagne. Ils ne semblent pas non plus avoir correspondu par lettre, mais chacun d'eux connaissait les publications de l'autre, et ils se citaient mutuellement avec beaucoup d'estime. Autour des années 1908/1910, Weber et Rostovtzeff comptaient l'un et l'autre parmi les meilleurs connaisseurs de l'histoire agraire de l'Antiquité. Néanmoins, ni les antiquisants ni les spécialistes d'historiographie ne se sont jamais vraiment intéressés aux rapports intellectuels et aux influences réciproques entre ces deux savants, moins encore à une véritable comparaison de leurs apports respectifs à l'histoire écono-

Version remaniée d'une communication présentée au colloque international "Mikhail I. Rostovtzeff", Maison des sciences de l'homme, Paris, 17-19 mai 2000. Cf., pour des développements ultérieurs : H. BRUHNS, "Max Weber und Michael I. Rostovtzeff. Oder : braucht (antike) Wirtschaftsgeschichte (moderne) ökonomische Theorie ?", in Europa und die Welt. Festschrift zum 60. Geburtstag von Dieter Berg, herausgegeben von R. AvERKORN, W. EBerhard, R. HaAs und B. SCHMies, Bochum, 2004, p. 150-171; et également : H. BRUHNS, "L'histoire économique de l'Antiquité a-t-elle besoin de la théorie économique? Interrogations à partir de l'œuvre de M.I. Rostovtzeff", Mediterraneo Antico VI/2 (2003) p. 571-595. 
mique de l'Antiquité. Les destins si différents et la chronologie décalée de leurs œuvres avaient de toute évidence créé une distance trop grande pour qu'une telle comparaison pût paraître légitime et pertinente. L'espace réduit de cet article nous permettra tout au plus de proposer une première esquisse de cette question. Nous commençerons par un rappel sommaire des rapports entre Weber et Rostovtzeff et nous nous arrêterons ensuite sur deux aspects de cette histoire : la question du colonat et leurs visions respectives du rapport entre histoire et théorie économique.

Max Weber s'est intéressé aux travaux de Rostovtzeff au plus tard à l'époque où il rédigeait la troisème édition des Agrarverhältnisse im Altertum pour le Handwörterbuch der Staatswissenschaften, c'est-à-dire entre octobre 1907 et février $1908{ }^{1}$. Rostovtzeff est cité ou mentionné huit fois dans les Agrarverhältnisse im Altertum; à chacune de ces mentions, Weber insiste sur l'importance des travaux de Rostovtzeff ou sur le fait que c'est Rostovtzeff qui, le premier, a relevé tel ou tel aspect essentiel ${ }^{2}$. Weber se réfere aux publications suivantes de l'historien russe :

- Geschichte der Staatspacht in der römischen Kaiserzeit (1902) [édition russe : 1899] ;

- Der Ursprung des Kolonats (1901) ${ }^{3}$;

- Kornerhebung und -transport im griechisch-römischen Ägypten (1906) ${ }^{4}$.

On peut illustrer ce premier constat par une ou deux citations qui témoignent de l'estime dans laquelle Weber tenait le savant russe et qui montrent de quelle façon il intégrait les résultats des recherches de Rostovtzeff dans sa propre analyse de l'économie antique. Par exemple à propos du

« système de liturgie [durant la période hellénistique] qui s'étend [...] à l'instrument le plus important de la constitution des fortunes capitalistes : la ferme d'État, et particulièrement la ferme fiscale (ce que Rostovtzeff a bien montré) ${ }^{5}$. "

Ou bien à propos de la cessation de l'afflux d'esclaves à bas prix vers les marchés et de la conquête de terres nouvelles susceptibles d'une exploitation capitaliste :

1 M. WEBER, “Agrarverhältnisse im Altertum”, in Handwörterbuch der Staatswissenschaften, 3. gänzlich umgearbeitete Auflage, t. 1, Jena, 1909, p. 52-188, réédité in M. WEBER, Gesammelte Aufsätze zur Sozial- und Wirtschaftsgeschichte [désormais cité GASW], édités par Marianne WebER, Tübingen, 1924, 2e édition 1988, p. 1-288). Pour l'histoire de ce texte et sa réception, voir l'introduction de $\mathrm{H}$. BRUHNS à la traduction française : “À propos de l'histoire ancienne et de l'économie politique chez Max Weber”, Introduction à M. Weber, Économie et société dans l'Antiquité, Paris, 1998 (réimpr. en coll. poche 2001), p. 9-59.

2 Ibidem, p. 116, 271, 274, 389, 392, 402, 403 deux fois. (= GASW, p. 30, 164, 167, 276, 279, 286 trois fois).

3 Daté par Weber par erreur de 1902.

4 Daté par Weber par erreur de 1904.

5 WeBER, Économie et société dans l'Antiquité, p. 274 (GASW, p. 167). 
"Ces phénomènes induisaient une stagnation et une régression du procès de formation du capital, accompagnées régulièrement de la tendance (récemment relevée avec beaucoup de pertinence par Rostovtzeff) à assurer la couverture des besoins publics par la différenciation et l'élargissement croissants du cercle des garants éventuels des prestations publiques par leur propriété ou leur qualification personnelle, et qui se trouvaient ainsi liés administrativement à leur fonction sociale et à leur propriété. Au bout du compte, on aboutit à cette domination universelle des liturgies et des munera, à cet anéantissement de tout ce que l'on avait nommé "liberté " aux époques "classiques " de l'Antiquité, et qui est caractéristique des "époques de déclin " des États antiques ${ }^{6}$."

Arnaldo Momigliano a insisté sur la grande influence que Mikhail Rostovtzeff, au même titre qu'Ulrich Wilcken, a exercée sur la partie des Agrarverhältnisse im Altertum consacrée à l'époque hellénistique. Et il résume, à ce propos, en une phrase la relation intellectuelle entre Weber et Rostovtzeff telle qu'il la conçoit :

"Con Rostovzev comincia il giuoco delle reciproche influenze, perché Rostovtzev fu sempre attento alle idee e ai metodi di Weber ${ }^{7}$."

Cette affirmation risque de nous entraîner tout simplement sur une fausse piste. La réception de Rostovtzeff par Weber est, par la force des choses, une histoire courte : après 1914, année au cours de laquelle il interrompit la rédaction de Die Stadt ${ }^{8}$, Weber n’a plus travaillé sur l'histoire ancienne proprement dite. Certes, des références nombreuses à l'histoire de l'Antiquité sont disséminées dans ses écrits ultérieurs, notamment dans Wirtschaft und Gesellschaft, mais sa dernière publication en tant que "spécialiste de l'Antiquité " date de 1909. Et surtout : Weber est décédé avant que Rostovtzeff ne publie les grandes histoires sociales et économiques qui l'ont rendu célèbre.

La réception de Weber par Rostovtzeff s'étend sur une période plus longue, et elle est apparemment plus complexe. On constatera d'abord qu'en 1910, dans ses travaux sur le colonat, Rostovtzeff cite Weber comme une grande autorité en la matière. Nous allons y revenir. À propos de la place de Weber dans les travaux ultérieurs de Rostovtzeff, en particulier dans les grandes histoires économiques et sociales de l'Empire romain et du monde hellénistique, nous avons le choix entre deux lectures. La

6 WEBER, Économie et société dans l'Antiquité, p. 116 (GASW, p. 30).

7 A. Momigliano, "Max Weber di fronte agli storici dell'antichità", in Settimo contributo alla storia degli studi classici e del mondo antico, Rome, 1984, p. 245-251, en part. p. 249.

8 Cf. H. BruHns, "Webers "Stadt" und die Stadtsoziologie", in H. BruHnS \& W. NiPPEL (éd.), Max Weber und die Stadt im Kulturvergleich, Göttingen, 2000, p. 39-62 ; ID., "La ville bourgeoise et l'émergence du capitalisme moderne : Max Weber : Die Stadt (1913/14 -1921)", in B. LepeTit \& C. TOpalov (éd.), La ville des sciences sociales, Paris, 2001, p. 47$78,315-319$ et $344-350$. 
première, que nous proposent certains auteurs, consisterait à dire que Rostovtzeff a traité souvent les mêmes thèmes et les mêmes questions que Weber, et parfois de la même manière, sans toutefois se référer expressément à lui. Vu leurs domaines d'intérêt, qui se recoupent largement, un tel constat n'est pas vraiment surprenant et ne constitue pas une grande révélation.

Une deuxième lecture, très différente, pourrait se fonder sur un inventaire des références explicites à Weber dans les travaux de Rostovtzeff. Celles-ci sont en fait très rares et, à les regarder de près, la conclusion la plus évidente est, qu'à cette époque, Rostovtzeff a, en fait, cessé de lire Weber, ou, du moins, n’a pas jugé nécessaire de le lire attentivement. On peut même s'interroger sur la façon dont il l'avait lu avant la guerre. Nous y reviendrons dans la dernière partie de cet article. Ce constat négatif ne fait d'ailleurs que confirmer le bilan qu'Alfred Heuss a dressé à l'occasion du centenaire de la naissance de Weber, bilan qui tenait en une seule phrase : les sciences de l'Antiquité ont fait leur chemin comme si Weber n'avait jamais existé ${ }^{9}$. Rostovtzeff ne constitue pas une exception à cet égard et, dans ces conditions, il serait vraiment surprenant que des antiquisants se seraient intéressés sérieusement à une comparaison entre Weber et Rostovtzeff, ou mieux entre leurs visions de l'économie antique.

À ma connaissance, il n'existe que deux tentatives de comparaison systématique entre Weber et Rostovtzeff ; toutes deux sont des travaux d'économistes datant du début des années 1930 et publiés dans des revues d'économie. Il s'agit d'Otto Zwiedineck, "Was macht ein Zeitalter kapitalistisch ?", paru dans la Zeitschrift für die gesamte Staatswissenschaft en 1931, et de Günther Wollheim, "Aufstieg und Niedergang des Kapitalismus im Römerreich nach Max Weber und Michael Rostovtzeff", publié dans les Jahrbücher für Nationalökonomie und Statistik en 1933. Certes, dans un certain nombre de travaux consacrés à Rostovtzeff, on trouve tel ou tel passage où, sur un point particulier, les positions de Rostovtzeff et de Weber sont comparées entre elles. Mais il ne s'agit là que de comparaisons ou de mises en relation ponctuelles. Ceci est particulièrement vrai pour les ouvrages consacrés aux théories du déclin de l'Empire romain. Weber et Rostovtzeff y figurent généralement en bonne place, mais même dans un livre comme celui de Santo Mazzarino, ou dans l'étude plus récente d'Alexander Demandt 10 , on chercherait en vain une analyse comparative des deux auteurs. Mazzarino se contente de faire état de la " critique radicalement négative " de Rostovtzeff à l'encontre de l'essai de Weber sur "Die sozialen Gründe des Untergangs

9 A. Heuss, "Max Webers Bedeutung für die Geschichte des griechisch-römischen Altertums", Historische Zeitschrift 201 (1965), p. 529-556, en part. p. 554 ; BRUHNS, "À propos de l'histoire ancienne", p. 11.

10 A. Demandt, Der Fall Roms. Die Auflösung des römischen Reiches im Urteil der Nachwelt, Munich, 1984, p. 290 ; S. MAZZARINO, La fin du monde antique. Avatars d'un thème historiographique, Paris, 1973 (éd. orig. 1959), p. 145. 
der antiken Kultur 11" (1896), l'œuvre, selon lui, " la plus géniale et la plus fondamentale qui ait été écrite sur la crise économique de l'Antiquité ». Demandt, de son côté, s'intéresse surtout à la question de savoir dans quelle mesure la lecture des travaux de Rostovtzeff a pu inciter Weber à modifier - partiellement - son interprétation de la crise dans les Agrarverhältnisse de 1909.

Les deux articles des années 1930, cités ci-dessus, se situent dans un contexte où le souvenir de l'École historique allemande de l'économie nationale n'avait pas encore complètement disparu et où un dialogue entre économistes et historiens sur l'économie antique n'était pas davantage considéré comme une idée exotique. Zwiedineck, auteur d'un important ouvrage sur la politique sociale ${ }^{12}$, publie son article dans la Zeitschrift für die gesamte Staatswissenschaft, qui porte aujourd'hui le titre de JITE. Journal of Institutional and Theoretic Economics. Il y dénonce l'utilisation, abusive selon lui, de l'épithète " capitaliste " par de nombreux historiens de l'économie antique. Le conflit entre «modernistes » et " primitivistes » n'est évidemment pas loin, et Zwiedineck termine d'ailleurs son article par une défense de Karl Bücher attaqué par certains historiens. Mais là n'est pas l'essentiel.

À la question de savoir si une époque historique comme l'Antiquité a été capitaliste ou non, Zwiedineck donne une double réponse. Il affirme d'abord qu'une telle question n'a aucune importance pour un économiste, mais il poursuit néanmoins : la façon dont certains historiens de l'économie traitent l'économie antique permet de vérifier si le travail conceptuel des économistes (nationalökonomische Begriffsbildung) est adéquat. Si tel est le cas, les concepts formés par les économistes doivent logiquement permettre aux historiens de "parvenir à des jugements clairs et transparents sur la réalité économique du passé "(p. 483). Au terme d'une analyse de l'utilisation des termes de "capital » et de "capitalisme » chez des historiens de l'Antiquité, en particulier dans la Social and Economic History of the Roman Empire de Rostovtzeff 13, Zwiedineck répond négativement aux deux questions qu'il s'était posées : les concepts utilisés par les économistes ne sont pas adéquats et les historiens n'arrivent pas à sortir du brouillard qu'ils répandent autour d'eux-mêmes en utilisant des concepts inappro-

11 La traduction française ( Les causes sociales du déclin de la civilisation antique ») figure dans WeBER, Économie et société dans l'Antiquité, p. 61-83.

12 O. ZWIEDINECK-SÜDENHORST, Sozialpolitik, Leipzig, 1911. L'auteur exploite les Agrarverhältnisse im Altertum pour les chapitres consacrés à l'Antiquité. Cf. le commentaire élogieux chez E. SALIN, Politische Ökonomie. Geschichte der wirtschaftspolitischen Ideen von Platon bis zur Gegenwart (fünfte erweiterte Auflage der Geschichte der Volkswirtschaftslehre), Tübingen - Zürich, 1967, p. 187.

13 M. Rostovtzeff, The Social and Economic History of the Roman Empire, Oxford, 1926, 2nd. ed. revised by P.M. FraSER, Oxford, 1957. Voir à présent l'édition italienne enrichie : M. RostovtzeFF, Storia economica e sociale dell'impero romano, Nuova edizione accresciuta di testi inediti a cura di Arnaldo MARCONE, Milan, 2003. Cf. aussi note 19. 
priés. Puis, s'appuyant largement sur Weber, Zwiedineck propose des définitions et des classifications susceptibles, selon lui, de répondre aux exigences qu'il avait formulées. La réaction immédiate de Rostovtzeff à la critique de Zwiedineck fut d'affirmer que "ce dernier défenseur de la théorie de Bücher " n’avait pas réussi à le convaincre. Et face à l'argumentation de l'économiste, Rostovtzeff a recours à l'ultime défense de l'historien ; contre " les mots et les concepts " il brandit " les faits " : " Doch handelt es sich nicht um Worte und Begriffe, sondern um Tatsachen ${ }^{14}$."

Larticle de Wollheim, publié deux ans après celui de Zwiedineck, en 1933, procède autrement. Au centre, de nouveau, le capitalisme. Mais l'auteur passe très rapidement sur les différences de conceptualisation chez Rostovtzeff et Weber (p. 390) ; il rappelle quelques traits du capitalisme antique chez Weber : capitalisme pillard ("Raubkapitalismus »), capitalisme politique, capitalisme côtier. Wollheim se contente ensuite d'affirmer que, chez Rostovtzeff, « l'idée du système économique n’apparait pas de façon aussi prononcée " que chez Weber. Après un résumé de leurs conceptions respectives et de tout ce qui leur est commun, résumé relativement court pour Weber, beaucoup plus long pour Rostovtzeff, l'auteur se concentre sur la vision si divergente que Weber et Rostovtzeff avaient des deux premiers siècles de notre ère. On pourrait résumer l'argumentation de Wollheim très grossièrement de la façon suivante : Weber se demandait pour quelle raison cette époque de paix n'avait pas connu une économie capitaliste, tandis que Rostovtzeff y voyait une époque de grande prospérité, caractérisée par l'existence de "capitalistes de grande envergure " (p. 391). À la différence de Zwiedineck, Wollheim n'insiste pas sur la nécessité d'utiliser des concepts clairs. Certes, il reproche à Rostovtzeff, par exemple, de présenter la théorie de l'oikos dans des termes complètement dépassés (p. 411), mais, pour prendre un autre exemple, la différence chez Weber et Rostovtzeff dans l'utilisation du concept d' " usine " (Fabrik) 15, appliqué à l'Antiquité, n'est à ses yeux qu'une querelle de mots. Weber, en effet, aurait associé à ce terme l'idée d'une bedeutende Apparatur (un important appareillage), tandis que Rostovtzeff aurait précisé que l'industrie antique ne connaissait pas de machines. Wollheim conclut sa lecture un peu trop rapide en disant : "De façon étrange, c'est justement le jugement que porte Rostovtzeff sur l'industrie qui recouvre en grande

14 Dans son compte rendu du livre de J. HASEBROEK, Griechische Wirtschafts- und Gesellschaftsgeschichte, également paru dans la Zeitschrift für die gesamte Staatswissenschaft 92 (1932), p. 333-339, en part. p. 334, n. 1 (réimpr. dans M. RostovTZEFF, Scripta Varia. Ellenismo e impero romano, éd. A. MARCONE, Bari, 1995).

15 Cf. tous les arguments que WEBER, Économie et société dans l'Antiquité, p. 106 sv., et passim (GASW, p. 8 sv.) avance contre l'emploi du terme " usine " par Eduard Meyer, ainsi que tous ses développements sur la différence des formes d'entreprise dans l'Antiquité, au Moyen Âge et à l'époque moderne. 
partie la vision qu'en avait Max Weber ${ }^{16}$. " Sa conclusion générale insiste davantage encore sur la proximité entre Weber et Rostovtzeff : «Au total, l'œuvre de Rostovtzeff peut remarquablement compléter la conception qu'a Max Weber du capitalisme antique, voire la remplacer dans ses traits principaux ${ }^{17}$. " Et il cite une phrase qu'il a probablement entendu prononcer par Weber dans un de ses cours à l'Université de Munich après la guerre : "À quoi sert, pour l'architecte le plus doué, son art [...], s'il ne dispose que d'un matériau insuffisant 18 ? " Toutes les différences entre Weber et Rostovtzeff s'expliqueraient donc uniquement par le matériau, les sources et les documents dont ils disposaient (ou ne disposaient pas) respectivement.

Zwiedineck et Wollheim envisagent ainsi, de façon très différente, les rapports entre l'œuvre de Weber et celle de Rostovtzeff. Sur un point essentiel, celui de l'analyse du système économique de l'Antiquité romaine, leurs visions sont même diamétralement opposées. Question de méthode ? Certainement. Avant d'y venir, nous devons faire un bref retour en arrière, à l'époque qui précède la guerre.

\section{Une rencontre autour de la question du colonat}

Les chemins de Weber et de Rostovtzeff se croisent à l'occasion de leur collaboration à la troisème édition du Handwörterbuch der Staatswissenschaften (HdWSt). Pour le premier tome, Weber a livré son copieux article Agrarverhältnisse im Altertum en février ou mars 1908 ; pour le tome V, contenant la lettre " $\mathrm{K}$ », qui doit paraître un an plus tard, il a promis un article sur le "Kolonat". Dans son introduction à la traduction française de la Social and Economic History of the Roman Empire, Jean Andreau rappelle que Weber a finalement décidé de céder cet article à Rostovtzeff 19 ; il évoque également le fait que la version russe de cette contribution de Rostovtzeff au HdWSt a été publiée en annexe à la traduction russe des Agrarverhältnisse de Weber, en $1925^{20}$, et cela certai-

16 G. Wollheim, "Aufstieg und Niedergang des Kapitalismus im Römerreich nach Max Weber und Michael Rostovtzeff", Jahrbücher für Nationalökonomie und Statistik 138 (1933), p. 390-412, en part. p. 411 : «Seltsamerweise deckt sich Rostovtzeffs Beurteilung gerade der Industrie in weitem Maße mit Max Webers Ansicht. "

17 Ibidem, p. 411 : "Im ganzen gesehen ist Rostovtzeffs Werk dazu angetan, Max Webers Auffassung von dem antiken Kapitalismus beträchtlich zu ergänzen, sie in der Hauptsache vielleicht sogar zu ersetzen. »

18 Ibidem, p. 411 : «Was nützt dem besten Architekten seine Kunst [...],, wenn ihm nur mangelhaftes Material zur Verfügung steht. »

19 J. ANDREAU, "Introduction" à M.I. ROSTOVTSEFF, Histoire économique et sociale de l'empire romain, Paris, 1988, p. I-LXXXIV, en part. p. XXI. Cf. M. RosTOWZEW (= RosTOVTSEFF), "Kolonat (Rom)", in Handwörterbuch der Staatswissenschaften, 3e édition, 1910, vol. 5, p. 913-921, réimpr. dans RostovTZEFF, Scripta Varia.

ANDREAU, "Introduction" (bibliographie de Rostovtzeff), p. 664. 
nement pas sans l'assentiment de Rostvtzeff, comme s'il fallait considérer son article "Kolonat" comme une suite directe de l'article Agrarverhältnisse de Weber. Deux questions se posent dès lors : à quel moment et pour quelle raison Weber a-t-il invité Rostovtzeff à rédiger à sa place l'article sur le colonat ? dans quelle mesure l'article "Kolonat" de Rostovtzeff correspond-il à celui que Weber avait songé à écrire luimême?

En vérité, nous ne disposons d'aucun témoignage qui permette d'affirmer que c'est Weber lui-même qui a proposé Rostovtzeff comme auteur pouvant le substituer. Pourtant, cette hypothèse n'est pas du tout exclue, vu les circonstances et le moment auquel Weber a renoncé à rédiger l'article "Kolonat". Weber avait, en effet, terminé la rédaction des Agrarverhältnisse au début de 1908, après quatre mois de travail proprement surhumain. Dès le mois de mai de la même année, l'article fut imprimé et diffusé comme cahier séparé, avant même la publication du volume correspondant du HdWSt. Dans une lettre du 22 mai 1908, Weber précise que l'article "Kolonat" "doit paraître l'année prochaine 21 ". Ce témoignage est important dans la mesure où il contribue à invalider la communis opinio des sociologues et weberologues qui considèrent que le retour de Weber à l'histoire ancienne en 1908, après une interruption de dix ans, n'avait été qu'un retour de circonstances, auquel Weber aurait consenti à la seule demande des éditeurs du HdWSt, pour mettre à jour son article Agrarverhältnisse im Altertum dont la deuxième édition remontait à l'année 1898. Si cela avait été le cas, pourquoi Weber aurait-il non seulement enrichi l'article de 1898 au point d'en faire un véritable livre au lieu de le raccourcir, comme on le lui avait demandé - mais aussi projeté une nouvelle étude sur l'Antiquité ? Dans ses lettres de l'hiver 1907/1908, Weber se plaint sans cesse de la corvée qu'était pour lui ce "foutu " article Agrargeschichte (Altertum). Et pourtant, ce calvaire de quatre mois ne le pousse pas à renoncer au projet d'article sur le colonat, en dépit du fait qu'il se plaint de sa situation : "La chose [les Agrarverhältnisse] est finie, et moi aussi ». «[...] la chose ne m'a procuré aucun plaisir (l'Antiquité est actuellement très loin de moi, j'ai dû absorber des masses immenses de publications nouvelles, car je n'avais rien lu depuis douze ans), elle m’a causé beaucoup de mal et, depuis novembre, je n'ai pu avoir une seule nuit de sommeil naturel ${ }^{22}$."

Malgré cela, il veut donc donner une suite à ce " foutu article ", et c'est très probablement à cause d'autres soucis et de nouveaux projets qu'il abandonnera finalement l'idée d'écrire l'article sur le colonat. Au mois d'octobre 1907, Max et Alfred Weber avaient proposé au Verein für Sozialpolitik d'entreprendre une série d'enquêtes sur le thème : Auslese und Anpassung der Arbeiterschaft in der geschlossenen Großindustrie

21 M. WebER, Briefe 1906-1908, in Max Weber Gesamtausgabe (désormais MWG), vol. II/5, Tübingen, 1990, p. 576.

22 Cf. BRUHns, "Introduction", p. 28. Lettre du 4 février 1908 à Marie Braun (MWG II/5, p. 430) ; cf. la lettre du même jour à Robert Michels, ainsi que celle du 24 mars, adressée également à Robert Michels. 
(Sélection et adaptation des ouvriers dans la grande industrie). Ce projet ayant été finalisé par un comité du Verein en juin 1908, Weber consacra les mois qui suivirent à la préparation de l'enquête. Durant l'été 1908, il rédigea une longue introduction méthodologique pour les futurs collaborateurs de l'enquête et dressa un état de la recherche sur la psycho-physique du travail industriel, qu'il compléta par une contribution fondée sur une enquête menée par lui-même, en septembre et octobre 1908, dans une usine de textile appartenant à des parents de sa femme. Il publia le tout en quatre articles dans Archiv für Sozialwissenschaft und Sozialpolitik en 1908 et 1909 23. À côté d'autres projets et travaux de publication, notamment le travail éditorial pour l'Archiv für Sozialwissenschaft und Sozialpolitik, il faut mentionner les préparatifs pour le Handbuch der politischen Ökonomie, qui deviendra par la suite le Grundriß der Sozialökonomie et, à partir du début de l'année 1909, sa participation de plus en plus centrale à la fondation de la Deutsche Gesellschaft für Soziologie. À partir de l'automne 1908, Weber intervient également, à plusieurs reprises, publiquement, dans la politique universitaire, ce qui entraîne une série de controverses à coup d'articles dans la presse ${ }^{24}$.

Après le mois de mai 1908, nous n'entendons plus Weber parler de l'article "Kolonat". Au mois d'août de la même année, Rostovtzeff participe au Congrès international d'histoire qui se tient à Berlin ${ }^{25}$. Il y prononce une conférence très remarquée sur le colonat ; on peut supposer que c'est vers ce moment que Weber céda l'article "Kolonat" à Rostovtzeff, éventuellement en suivant là une suggestion d'Eduard Meyer qui faisait partie du comité organisateur du Congrès auquel, d'ailleurs, Weber luimême n'avait pas participé 26 .

Que peut-on dire de l'article "Kolonat" que Weber projetait encore d'écrire au mois de mai 1908 ? Dans les Agrarverhältnisse im Altertum, Weber renvoie le lecteur au moins neuf fois à l'article "Kolonat" qui était censé suivre ${ }^{27}$. Ces renvois montrent

23 Pour la chronologie, voir l'introduction à $M W G$ II / 5, p. 3 sv.

24 Voir le choix de textes publiés en français dans M. WEBER, De la liberté intellectuelle et de la dignité de la vocation universitaire. Écrits de Max Weber. Édités et traduits par M.L. MARTIN, Toulouse, 1983.

25 Voir K.-D. ERDMANN, Die Ökumene der Historiker. Geschichte der internationalen Historikerkongresse und des Comité International des Sciences Historiques, Göttingen, 1967, p. 83. C'est Wilamowitz qui avait demandé à Rostovtzeff de prononcer une des deux grandes conférences réservées aux sciences de l'Antiquité lors du congrès de 1908. Cf. sa lettre à Franz Cumont, datée du 1 juin 1907, dans laquelle il propose à ce dernier l'autre grande conférence : cf. C. BONNET, Le "grand atelier de la science». Franz Cumont et l'Altertumswissenschaft. Héritages et émancipations. I. Des études universitaires à la fin de la Première Guerre mondiale, 1888-1923 (Études de philologie, d'archéologie et d'histoire ancienne de l'Institut historique belge de Rome), Bruxelles-Rome, 2005 (sous presse). Les lettres de Weber montrent qu'il se trouvait à Heidelberg à l'époque du Congrès.

Certains de ces renvois ont été supprimés par Marianne Weber lors de la réédition du texte dans GASW en 1924. Ils ont été rétablis par H. Bruhns dans l'édition française : WeBER, Économie et société dans l'Antiquité, p. 128, 177, 273, 276, 358, 385, 387, 390, 403. 
d'abord que, tout au long de son travail, Weber a considéré ce deuxième article comme une suite naturelle des Agrarverhältnisse, suite sur laquelle il anticipe parfois, et dans laquelle il voulait reprendre et approfondir certaines questions. En ce sens, l'édition russe qui juxtapose les Agrarverhältnisse de Weber et le Kolonat de Rostovtzeff suit à la lettre les intentions du premier. Peut-on dire la même chose du point de vue du contenu de l'article de Rostovtzeff ? Nous allons y venir. Les renvois de Weber à l'article "Kolonat" tel qu'il l'avait projeté nous fournissent quelques indications sur l'orientation qu'il pensait lui donner :

- l'article traitera des phénomènes centraux de l'histoire agraire de l'empire romain et de la seigneurie de l'Antiquité tardive en général (p. 128);

- concernant le recours, pour les besoins de l'État, à l'économie monétaire, recours qui selon Weber fut le levier de la transformation de l'économie tout entière dans le sens d'une dépendance croissante de l'économie communautaire et d'une élimination des échanges, il veut analyser en détail l'État liturgique, pour lequel l'Égypte représente à ses yeux l'État modèle de la période hellénistique (p. 273, p. 387);

- à l'époque de l'expansion romaine, l'administration de l'aristocratie romaine a partout favorisé la naissance de grandes propriétés foncières. Ce processus sera analysé sous l'aspect politique (Rome aide les oligarchies à accéder au pouvoir et s'appuie sur elles) et sous l'aspect juridique : le mode de disposition du sol et le droit foncier (p. 358) ;

- par rapport aux Agrarverhältnisse, Weber veut approfondir, dans ce deuxième article, l'analyse économique du processus à travers lequel les grandes propriétés foncières continuent à s'étendre en se détachant progressivement du marché, de moins en moins nécessaire pour combler leurs besoins (p. 385) ;

- l'article "Kolonat" sera l'occasion de réfléchir sur le développement de la civilisation européenne méditerranéenne, qui ne suit ni des cycles clos ni une évolution linéaire : "Le développement de la civilisation européenne méditerranéenne n’a connu jusqu’à présent ni "cycles" clos, ni une évolution linéaire clairement orientée. Des phénomènes pour un temps totalement enfouis de la civilisation antique ont resurgi parfois dans un monde qui leur était étranger. D'autre part, de même que les villes de l'Antiquité tardive, notamment les villes hellénistiques, préparent le Moyen Âge dans le domaine de l'industrie [Gewerbe], de même les seigneuries foncières de l'Antiquité tardive le font dans le domaine agraire. La validité et les limites de cette affirmation seront discutées en un autre lieu (cf. l'article “Kolonat”) " (p. 390);

- dans la lettre, déjà citée, du 22 mai 1908 à Ulrich Stutz, l'un des éditeurs de la Zeitschrift der Savignystiftung für Rechtsgeschichte, à qui il avait envoyé un tiré-à-part des Agrarverhältnisse, Weber écrit : "Vous aurez vu dans mon envoi que l'analyse de la seigneurie foncière antique est encore devant moi, que je n'y arrive que maintenant. Et, en liaison avec cela, je veux reprendre toutes ces idées et toute cette problématique. [...] L'article "Colonat" doit paraitre l'année prochaine. Puisque je veux y aborder, pour la 
comparaison, la seigneurie foncière en général, je dois alors entrer dans l'histoire du droit allemand ${ }^{28}$. "

Ces quelques traits esquissés rapidement font apparaître un projet qui devait embrasser la question de la seigneurie foncière depuis l'Orient ancien jusqu'au Moyen Âge allemand. Pour cela, Weber aurait évidemment pu s'appuyer sur certains de ses travaux antérieurs : la Römische Agrargeschichte, les Agrarverhältnisse im Altertum, et l'article de 1904 sur Der Streit um den Charakter der altgermanischen Sozialverfassung in der deutschen Literatur des letzten Jahrzehnts. Mais pour aller aussi loin qu'il l'annonçait dans la lettre à Stutz, il aurait dû accomplir un effort comparable à celui qu'il avait entrepris pour les Agrarverhältnisse im Altertum. En regard des autres grands projets mis en chantier en 1909 et 1910, c'était totalement irréaliste, même pour un Max Weber. Il lui fallait y renoncer, au moins provisoirememt.

L'article rédigé par Rostovtzeff à la place de Weber pour le Handwörterbuch est d'une taille modeste. Pour la dimension de sa contribution, Rostovtzeff s'est, de toute évidence, conformé aux instructions données par les éditeurs, instructions que Weber avait superbement ignorées pour les Agrarverhältnisse im Altertum. Pour une comparaison avec le projet de Weber, il faut par conséquent prendre également en compte les Studien zur Geschichte des römischen Kolonats de Rostovtzeff, parues la même année (1910) que l'article "Kolonat". Alfred Heuss (1965) et, plus récemment, Arnaldo Marcone (1988) ont insisté sur la surprenante convergence entre les Agrarverhältnisse im Altertum de Weber et le "Kolonat" de Rostovtzeff. Il n'est ni possible ni nécessaire de rappeler ici tous les points majeurs sur lesquels Weber et Rostovtzeff partagent les mêmes vues et la même approche. Dans les deux textes publiés en 1910, Rostovtzeff a clairement signalé cette convergence et ses dettes par rapport à Weber, comme celui-ci l'avait du reste fait dans les Agrarverhältnisse im Altertum par rapport aux travaux antérieurs de Rostovtzeff. Dans la préface de ses Studien, Rostovtzeff parle des « beaux résultats dans notre champ de recherche qu'a obtenu Max Weber dans son article révolutionnaire (bahnbrechend) "Agrargeschichte 29 " du Handwörterbuch der Staatswissenschaften. Dans le corps du livre, il le cite une dizaine de fois, dont une seule fois

28 Lettre du 22 mai 1908 à Ulrich Stutz, $M W G$ II/5, p. 576.

29 "Agrargeschichte" est le titre général donné aux trois contributions sur les "Agrarverhältnisse" dans l'Antiquité, le Moyen Âge et les temps modernes. Cf. les tableaux représentant la répartition des entrées " agraires " par rapport aux contributions de Weber dans les différentes éditions du Handwörterbuch der Staatswissenschaften dans H. BRUHNS (éd.), Histoire et économie politique en Allemagne de Gustav Schmoller à Max Weber. Nouvelles perspectives sur l'école historique de l'économie, Paris, 2004, p. 198-200, et dans H. BRUHNS \& J. ANDREAU (éd.), Sociologie économique et économie de l'Antiquité : à propos de Max Weber, Cahiers du Centre de recherches historiques, $\mathrm{n}^{\circ} 34$, octobre 2004, p. 37. 
en exprimant un point de vue différent de celui de Weber ${ }^{30}$. Cette grande proximité entre les deux savants repose, d'une part, sur leur connaissance intime des sources antiques et de la production scientifique dans tous les domaines concernés et, d'autre part, sur une approche analogue, très empirique, de l'histoire agraire de l'Antiquité, privilégiant souvent les mêmes questions, et accordant autant d'attention aux dimensions économiques que juridiques.

À côté de ces convergences, il existe une série de différences. Les lignes directrices de ce que Weber aurait voulu écrire dans l'article "Kolonat" apparaissent de façon relativement claire dans les Agrarverbältnisse im Altertum et dans la lettre à Stutz citée plus haut. Certaines perspectives de recherche annoncées par Weber sont complètement absentes des deux textes de Rostovtzeff, en particulier :

- l'élargissement de la question du colonat à une analyse générale de la seigneurie (Grundherrschaft) ;

- le passage de la seigneurie antique et du colonat aux structures agraires du Moyen Âge ;

- l'analyse du rapport entre grande propriété et marché.

Les démarches de Weber et de Rostovtzeff sont donc différentes. Si le premier cherche à étendre l'analyse des structures agraires à une comparaison avec d'autres époques, le second s'efforce d'élargir, à l'intérieur de ses propres domaines de recherche, l'histoire ancienne et l'archéologie, les bases documentaires pour les questions qui l'intéressent, d'abord le colonat, ensuite toute l'histoire économique de l'Empire romain. Les Agrarverhältnisse im Altertum sont, en revanche, une histoire sociale et économique générale de l'Antiquité, comme l'affirme Marianne Weber lors de la réédition du texte en $1924{ }^{31}$. Dans la biographie qu'elle consacrera peu de temps après à son mari, elle est peut-être encore plus près de la réalité : les Agrarverhältnisse sont, écrit-elle, " en réalité une sorte de sociologie de l'Antiquité, c'est-à-dire une analyse historique qui, à l'aide d'un travail conceptuel, réussit à pénétrer toutes les structures importantes de la vie sociale de l'Antiquité 32 ».

30 M. RostovtzefF, Studien zur Geschichte des römischen Kolonats (1. Beiheft zum Archiv für Patyrusforschung), Leipzig et Berlin, B.G. Teubner, p. VII, 133 n. 2, 259 n. 1, 306 n. 1, 314 n. 1, n. 2, 316 n. 1, 317 n. 1, 377 n. 1, 402.

31 GASW p. 1, n. 1.

32 Marianne Weber, Max Weber. Ein Lebensbild, Tübingen, Mohr 1926 (réimpr. Heidelberg, 1950), p. 343 sv. : " ... in Wahrheit eine Art Soziologie des Altertums, d.h. eine historische Analyse und begriffliche Durchdringung aller wichtigen Strukturformen des sozialen Lebens der Antike. Ein riesiges historisches Material ist hier in knappeste, präziseste Form zusammengepreßt. [...] Alle wichtigen sozialen Erscheinungen werden gegeneinander und gegen mittelalterliche und neuzeitliche gehalten, das eine durch das andere erklärt, das Typische und das Individuelle gesondert, am Gleichbenannten das Verschiedene gezeigt und vermittelst präziser "Idealtypen " veranschaulicht, wo es zu Irrtum führt, wenn der Historiker moderne Erscheinungen in die Vergangenheit hineininterpretiert. " 
On devrait pourtant y ajouter un point essentiel : si le plan - par périodes et par grandes entités politiques et historiques - des Agrarverhältnisse im Altertum se conforme au genre du Handwörterbuch, il en differe par une longue introduction, tout à fait inhabituelle, par laquelle Weber fait précéder l'ensemble. Cette introduction porte le titre de "Contribution à la théorie économique du monde des États antiques " et remplit trois fonctions :

- clarification des concepts utilisés et de la méthode de conceptualisation ;

- explicitation du principe structurant l'analyse de l'histoire agraire : une typologie des stades d'organisation urbaine, en raison de l'imbrication étroite entre histoire agraire et histoire urbaine dans l'Antiquité ;

- explicitation d'une problématique centrale : «Mais la question que nous nous posons est la suivante : l'Antiquité connaît-elle l'économie capitaliste, à un degré qui soit significatif du point de vue de l'histoire de la civilisation 33 ? "

Cette problématique amène Weber à adopter systématiquement une démarche comparative. Non pour chercher des analogies, comme Rostovtzeff le fait souvent, mais au contraire pour mieux faire ressortir les différences. Ce qui intéressait Weber dans son analyse du capitalisme comme système économique, c'étaient " les traits qui n’étaient pas présents sous cette forme à d'autres époques historiques 34 ". C'est certainement ainsi qu'il aurait également conçu son analyse du colonat, c'est-à-dire en intégrant l'histoire du colonat de l'Antiquité tardive dans une perspective de longue durée et dans une comparaison avec des phénomènes plus ou moins proches à d'autres époques et dans d'autres aires culturelles.

\section{Du bon et du mauvais usage de la théorie}

Après la guerre mondiale, la révolution russe, l'exil de Rostovtzeff, la mort de Weber, tout ce qui rapprochait l'historien russe de l'auteur des Agrarverhältnisse im Altertum semble oublié. Dans The Social and Economic History of the Roman Empire, Weber n'est cité qu'une seule fois, sous la rubrique, pour ainsi dire, de la fausse théorie. Dans The Social and Economic History of the Hellenistic World ${ }^{35}$, il n'apparaît plus du tout. La seule évocation de Weber dans The Social and Economic History of the Roman Empire, avec un simple renvoi à Wirtschaft und Gesellschaft, se trouve dans le chapitre sur les Flaviens et les Antonins, à propos de la question :

33 GASW, p. 12 : "Die Frage ist nun : kennt das Altertum (in einem kulturhistorisch relevanten Maß) kapitalistische Wirtschaft ?" ; WEBER, Économie et société dans l'Antiquité, p. 98.

34 Cf. l'introduction de H. BRUHNS à WEBER, Économie et société dans l'Antiquité, p. 44.

35 Parue à Oxford en 1941 : M.I. ROSTOVTSEFF, Histoire économique et sociale du monde hellénistique, Paris, 1989. 
"Pourquoi l'industrie antique n'atteignit-elle pas les sommets de développement qu'a connus le monde moderne ? Pourquoi l'industrialisation du monde antique s'est-elle interrompue, et pourquoi l'Empire romain n'est-il pas parvenu à mettre sur pied des formes industrielles capitalistiques caractéristiques de notre temps?"

Cette question, Rostovtzeff aurait pu la trouver, formulée dans les mêmes termes que nous venons de citer ci-dessus, dans les Agrarverhältnisse im Altertum. De même que dans un autre texte, publié après la mort de Weber, en 1921 et traduit dès 1923 en russe :

"Dans la période intermédiaire [c'est-à-dire entre l'époque carolingienne, d'une part, et l'État moderne patriomonial, d'autre part, deux époques où, dit Weber, les villes n'étaient pratiquement que des circonscriptions administratives, H.B.], elles (les villes) étaient à un certain degré partout des " communes " avec des droits politiques propres et une politique économique autonome. L'évolution dans l'Antiquité se déroula de manière comparable. Et pourtant, ni le capitalisme moderne ni l'État moderne n'ont poussé sur le sol des villes antiques, alors que le développement urbain médiéval, bien que n’ayant pas été la seule phase préliminaire décisive ni surtout le porteur du capitalisme et de l'État modernes, n’en a pas moins été un facteur absolument déterminant de leur naissance ${ }^{36}$. "

Dans les Agrarverhältnisse, Weber avait insisté longuement sur les différents obstacles à un développement capitaliste de l'économie antique 37 :

1. le caractère politique spécifique des communautés antiques, analysé en détail par Weber dans les pages précédentes;

2. la spécificité économique de l'Antiquité, c'est-à-dire :

- les limites de la production marchande, du fait des limites techniques du transport (économique) des biens de l'intérieur du pays vers l'extérieur et réciproquement ;

- l'instabilité constitutive et économiquement déterminée du capital existant et de la formation de capital ;

- la limite technique des possibilités d'exploitation du travail servile dans la grande entreprise ;

- les limites de la « précision comptable » (impossibilité d'un calcul rigoureux lorsqu’on utilise du travail servile);

36 M. Weber, Die Stadt, édité par Wilfried NipPEL, Tübingen 1999 (= MWG I, 22-5), p. 233, poursuit ainsi : "En dépit de toutes les similitudes externes de l'évolution, on doit, dans un second temps, constater aussi des différences profondes. C'est vers elles que nous nous tournerons maintenant. C'est en opposant les deux types de villes l'un à l'autre dans leurs formes caractéristiques que nous aurons la meilleure chance d'apercevoir ces différences. " (trad. par H.B. ; dans l'édition française [Max Weber, La ville, traduit de l'allemand par Philippe Fritsch, Paris 1982, p. 155] ce passage essentiel est tronqué et déformé). 
- le caractère spécifiquement instable de la " grande entreprise » : "Le facteur de l'unité des "grandes entreprises" esclavagistes de l'Antiquité n'est pas une contrainte objective, c'est-à-dire la division et la composition du travail liées au mode de production, il est purement personnel : l'accumulation fortuite de la possession d'hommes entre les mains d'un individu. C'est là le sens exact de la théorie de l'oikos" ;

- la position sociale précaire de l'entrepreneur capitaliste antique (" qu’il faut bien distinguer du rentier du capital »);

- absence de toute transfiguration éthique du travail accompli en vue d'un gain ;

- le caractère essentiellement discontinu de la disposition de la propriété du capital, au contraire d'une " entreprise » continue, par exemple dans les activités de négoce maritime des couches socialement respectables ;

- importance des différenciations statutaires de la population, et des différenciations politiquement déterminées du droit relatif à la fortune (en particulier du droit foncier et du droit successoral) qui pouvaient être avant tout des sources de rentes. D'où une prépondérance, dans les démocraties particulièrement, du point de vue petit-bourgeois des rentes et de l'intérêt " alimentaire ».

Mais au lieu de retourner à ce texte de Weber, qu'il connaît pourtant, Rostovtzeff affirme :

"La réponse proposée par d'éminents économistes modernes tels que K. Bücher, G. Salvioli et M. Weber, est que l'industrie fut incapable de se développer parce que le monde antique ne se détourna jamais des formes d'économie domestique (Oikenwirtschaft) primitive : il ne parvint jamais aux phases supérieures du développement économique atteintes à l'époque moderne - celle de l'économie urbaine et de l'économie nationale ${ }^{38}$."

Ni Bücher ni surtout Weber n'ont jamais dit chose pareille, et Salvioli peut-être non plus ${ }^{39}$. Devons-nous en conclure que Rostovtzeff n'a pas véritablement lu ces trois auteurs ? En vérité, les choses sont un peu plus compliquées. En 1932, dès la première phrase de son compte rendu de la Griechische Wirtschafts- und Gesellschaftsgeschichte de Johannes Hasebroek, Rostovtzeff place ce livre dans le contexte du conflit entre primitivistes et modernistes. En même temps, il tient à dissocier Weber de Bücher, en précisant que la célèbre théorie de K. Bücher avait été modifiée, dans un sens plus acceptable

38 M.I. RostovtsefF, Histoire économique et sociale de l'empire romain, Paris, p. 251 et note 107 (éd. orig. The Social and Economic History of the Roman Empire, Oxford, $1926 ; 2^{\mathrm{e}}$ éd. 1957, p. 349 et n. 107) ; Rostovtzeff se réfere aux pages du $\$ 7$, "Die Entwicklung zum Oikos", du chapitre III ("Typen der Vergemeinschaftung und Vergesellschaftung in ihrer Beziehung zur Wirtschaft") de la première partie de Wirtschaft und Gesellschaft (dans l'édition française : M. Weber, Économie et société, t. 1, Paris 1971, réimpr. en 2 vol. en 1995 dans la collection Agora Pocket, t. 2, p. 118-123).

39 Voir R. MEYER, "Historian of the classic world : a critique of Rostovtzeff", Science and Society 10 (1946), p. 361-391. 
pour les historiens, par le "génial M. Weber ". Il accuse ensuite Hasebroek d'avoir voulu sauver l'essentiel de la théorie de Bücher. Aux yeux de Rostovtzeff, cela rendait Hasebroek doublement coupable, car celui-ci suivait Bücher bien qu'étant " en pleine possession de la méthode historique » et bien qu'étant «armé de la totalité de l'équipement du philologue classique et de l'historien. " Weber, par contre, obtient un non-lieu dans ce procès, car il était « surtout philosophe et théoricien 40 ». Pas compétent, donc pas coupable, mais peut-être responsable.

Pratiquement à la même époque, on peut le lire encore autrement. En 1930, Rostovtzeff publie, dans la Economic History Review, le texte d'une conférence intitulée "The Decay of the Ancient World and its Economic Explanations 41 ». D'emblée, il insiste sur la difficulté du dialogue entre historiens de l'économie, d'une part, et économistes de l'autre, à propos de la question de savoir comment des théories économiques créées et diffusées par des économistes peuvent être appliquées à l'histoire. De nouveau, Rostovtzeff vise les fabricants de théories que sont à ses yeux Bücher, Salvioli, Weber et, à l'arrière-plan, Marx et Rodbertus. Pour notre propos, ce texte est intéressant à deux titres. D'abord en ce qui concerne Weber : contrairement à la Social and Economic History of the Roman Empire, où le lecteur n'avait été renvoyé qu'à un seul passage d'Économie et Société (Wirtschaft und Gesellschaft), Rostovtzeff se réfere ici de nouveau aux Agrarverhältnisse im Altertum ainsi qu’à l'essai sur "Die sozialen Gründe des Untergangs der antiken Kultur". Il se démarque des "brillantes et stimulantes conclusions " de Weber sur le caractère de la vie économique du monde ancien, car, selon lui, celles-ci reposent sur des fondements plutôt faibles et incomplets. Il ajoute que l'antithèse weberienne entre "ancient "homo politicus" and modern "homo oconomicus" " devrait sans doute être soigneusement prise en compte par les historiens du monde antique. Mais cette opposition ne pourrait s'appliquer :

"To those periods when political life was concentrated in the hands of a few, and the majority was absorbed by economic, intellectual and religious interests. »

Rostovtzeff s'était d'emblée placé sur le terrain conflictuel qui opposait historiens et économistes. Il avait, en effet, commencé son article par la phrase suivante :

"It is not an easy task for one who happens to be an historian and not an economist to talk to economists on theories which bear on economic history and have been created and put into circulation by economists. "

Face à ses lecteurs économistes, il annonce clairement ce qu'il entend par théories et concepts économiques. Théorie économique, c'est la théorie des stades d'évolution

40 Rostovtzeff, compte rendu de HASEBroeK, Griechische Wirtschafts- und Gesellschaftsgeschichte, p. 333 et 335.

41 M. RostovTzeFF, "The Decay of the ancient world and its economic explanations", Economic History Review 2 (1930), p. 197-214. Réimprimé dans RostovTZEFF, Scripta Varia, p. 215-230. 
économique, la fameuse Stufentheorie, théorie des stades économiques, qui alimentera encore pendant longtemps les débats sur la nature de l'économie antique. Selon Rostovtzeff, cette théorie débouche sur des affirmations qui ne sont rien d'autre que des constructions modernes : l'économie d'oikos " is a theory, a construction, not a reality " (p. 208/226). Très juste, et sans s'en rendre compte, il ne dit rien d'autre que ce qu’avaient souligné Bücher et Weber : la catégorie de "Oiken-Wirtschaft " ( économie d'oikos ») de Rodbertus est conçue par eux

"au sens d'une construction "idéaltypique" d'un mode d'organisation économique apparu dans l'Antiquité sous une forme particulièrement proche de la pureté conceptuelle, avec ses conséquences spécifiques, sans que pourtant l'Antiquité entière, dans toute son extension temporelle et spatiale, ait été dominée par ce mode d'organisation. Et l'on peut ajouter sans risque que cette "domination", durant les périodes où elle était effective, ne signifiait rien de plus qu'une restriction de l'importance des échanges pour la couverture des besoins [...], ainsi qu'un déclassement économique et social des couches qui auraient pu être porteuses de ces échanges $42 »$.

Rostovtzeff affiche son "innate dislike " pour la théorisation de l'histoire (p. 197/215) qui consiste, selon lui, à appliquer de la fausse théorie économique à l'histoire. La bonne analyse économique de l'histoire, à l'opposé, c'est celle qui analyse les différentes périodes et entités politiques en fonction de la part que prend l'État dans l'économie, ou en fonction de l'extension ou du rétrécissement du marché. Ou encore, pour prendre une autre distinction théorique chez Rostovtzeff : l'opposition entre, d'une part, la forme du commerce capitaliste moderne, fondé sur de grandes et riches compagnies commerciales, et, d'autre part, un univers des affaires totalement individualiste ${ }^{43}$. L'axiome fondamental de Rostovtzeff, celui de l'« absence of qualitative differences between the structure of ancient civilization and that of modern capitalist society 44 » rend en effet superflu tout effort pour élaborer des concepts susceptibles de faire apparaitre des différences qualitatives.

Il est difficile d'imaginer un contraste plus grand avec la pensée de Weber. Il serait tentant d'aller dans le détail et d'illustrer ce contraste à l'aide de quelques exemples, comme les distinctions conceptuelles fondamentales entre "fortune " et " capital ",

42 WeBER, Économie et société dans l'Antiquité, p. 93 (GASW, p. 7 sv.).

43 RostovtsefF, Histoire économique et sociale de l'empire romain, p. 135.

44 R. MeYer : "Historian of the Classic World", p. 362 sv., cite Rostovtzeff : "The modern development [...] differs from the ancient only in quantity and not in quality." Cf. aussi M. RostovtzefF, A History of the Ancient World, 2 éd., Oxford, 1929-30, vol. I, p. 10, cf. RostovtzefF, compte rendu de HaseBroecK, Griechische Wirtschafts- und Gesellschaftsgeschichte, p. 334, n. 1. La critique de Rostovtzeff par Reinhold Meyer compte parmi les plus pertinentes. Malheureusement il se contente de combattre Rostovtzeff avec les armes de Marx sans recourir à celles qu'il aurait pu trouver chez Weber qu'il n'a pas lu et au sujet duquel il se trompe (p. 387) de la même façon que Rostovtzeff. 
entre " Haushalt » (oikos) et " kontinuierlicher Erwerbsbetrieb " (entreprise fondée sur l'acquisition continuelle), entre les différents types de " chances de gain ", la nature de la répartition ou de la combinaison des prestations économiques, du point de vue technique, économique, ou par rapport au régime juridique de propriété ${ }^{45}$. Bref, tout ce qu'on trouve déjà dans les Agrarverhältnisse et que Weber développera plus tard dans sa sociologie économique ${ }^{46}$. Toutes ces distinctions sont gommées chez Rostovtzeff qui, au fond, confond théorie économique avec politique ou idéologie économique, et qui ne prend pas du tout en compte l'importante fonction instrumentale des concepts dans les travaux de Weber.

À la fin des Agrarverhältnisse, dans la bibliographie commentée, Weber s'était, en effet, adressé aux historiens :

"L'erreur principale que continuent de commettre nombre d'historiens - pas tous, néanmoins - vient de ce que la "complexité" et la "fluidité" des phénomènes historiques ne permettraient pas d'utiliser des concepts stables et précis. L'exemple suivant illustrera notre propos : il y a le petit artisan qui emploie régulièrement ou à l'occasion un esclave, mais participe lui-même au travail ; il y a ensuite l'artisan qui, tout en ayant été formé à son art, ne fait principalement que surveiller ses esclaves; puis il y a celui qui confie ce soin à l'un de ses esclaves, parfois, souvent ou toujours; enfin, le pur commerçant qui, ne maitrisant que peu ou pas du tout la technique, conduit ses affaires comme un "directeur commercial" ; ou encore celui qui ne fait valoriser par ses propres esclaves qu'une partie de la matière première ; puis le commerçant ou le particulier qui "investit" occasionnellement son argent dans un ou plusieurs esclaves formés à une technique; enfin la maison princière, qui fait travailler des esclaves qualifiés pour une production marchande, mais aussi pour ses besoins privés, ou, pour finir, seulement pour ses besoins privés : ces cas de figures constituent une chaîne ininterrompue de possibilités. Mais cette diversité inarticulée des faits ne justifie pas cependant que nous forgions des concepts imprécis : au contraire. Elle nous oblige à utiliser de façon appropriée des concepts précis ("idéaltypiques" : cf. Archiv für Sozialwissenschaft, XIX, 1) ${ }^{47}$, non comme des schémas qui conduiraient à faire violence aux données historiques, mais comme un moyen de définir le

45 Cf. H. Bruhns, "Max Weber, l'économie et l'histoire", Annales. Histoire, Sciences Sociales 51/6 (1996), p. 1259-1287, p. 1284.

46 Cf. surtout R. Swedberg, Max Weber and the Idea of Economic Sociology, Princeton, New Jersey, 1998.

47 Weber renvoie ici à son article de 1904 sur "Die 'Objektivität' sozialwissenschaftlicher und sozialpolitischer Erkenntnis", dans M. WeBER, Gesammelte Aufsätze zur Wissenschaftslehre, édités par J. WinCKELMANN, Tübingen, 7e éd. 1988, p. 146-214 [première publication : Archiv fir Sozialwissenschaft und Sozialpolitik, 19 (1904)], traduction française : "L'objectivité de la connaissance dans les sciences et la politique sociales", in Essais sur la théorie de la science, Paris, 1965, réimpr. coll. Agora, 1992, p. 117-201. 
caractère économique d'un phénomène : parvenir à déterminer dans quelle mesure il se rapproche de tel ou tel "type idéal”. Une brève esquisse du genre de celle-ci n'évite évidemment pas tout à fait le schématisme ${ }^{48}$."

La réaction à la fois d'attirance et de rejet, et au fond d'incompréhension, que Rostovtzeff manifestait à l'égard d'une opposition idéaltypique comme celle entre homo politicus et homo oconomicus (employée par Weber pour clarifier les différences entre les économies urbaines des cités autonomes antiques et certaines villes autonomes médiévales) n'est qu'un signe de son refus de se laisser entraîner dans une discussion sur la nature du travail des sciences sociales. Ce refus est également sous-tendu par une deuxième différence fondamentale avec Weber : le statut de la comparaison. Je cite, à ce propos, la dernière phrase des Agrarverhältnisse im Altertum (à nouveau dans le chapitre bibliographique) :

"On aurait bien besoin d'une comparaison vraiment critique des phases de développement de la cité antique et de la ville médiévale [...]. Pour être fructueuse, il faudrait cependant qu'elle ne cherche point des "analogies" et des "parallèles", comme ces schémas d'évolution généraux qu'il est de mode de construire aujourd'hui ; au contraire, elle devrait mettre en lumière la particularité de deux processus de développement qui aboutissent à des résultats si différents, et guider ainsi l'imputation causale de ce déroulement différent. Pour ce faire, il est indispensable d'isoler au préalable (i. e. d'abstraire) les composantes particulières des phénomènes; la comparaison demande ensuite que chaque composante particulière soit éclairée par des règles d'expérience et par la construction de concepts clairs (comme nous l'avons dit dans l'introduction), faute de quoi on ne peut parvenir à aucune imputation causale assurée : c'est une nécessité générale qui devrait être respectée notamment dans l'étude des phénomènes économiques, car un défaut de précision des concepts peut y entraîner les jugements les plus erronés que l'on puisse imaginer ${ }^{49}$."

La brève rencontre, autour de 1908/1910, entre Mikhail I. Rostovtzeff et Max Weber a ainsi été en grande partie une rencontre manquée. Une rencontre manquée de l'histoire avec l'économie, ou avec les sciences sociales, si l'on veut. Rostovtzeff a apprécié Weber en tant qu'érudit et spécialiste de l'histoire économique antique. Il a compris

48 WeBER, Économie et société dans l'Antiquité, p. 393 (GASW, p. 280).

49 WEBER, Économie et société dans l'Antiquité, p. 405 (GASW, p. 288). 
la stérilité de la querelle Bücher-Meyer, mais en refusant de lire les travaux du "génial » Weber au-delà de ce qui l'intéressait de façon immédiate, Rostovtzeff s'est privé de la possibilité de comprendre la position de Weber tant par rapport à ce débat, que par rapport aux théories et méthodes des économistes de son temps. Quand Rostovtzeff s'est adressé dans la Economic History Review expressément à un public d'économistes anglophones, il semble ne pas avoir envisagé le fait que les lecteurs de cette revue pourraient avoir une conception différente de ce qu'était la théorie économique ou de qui étaient les principaux théoriciens de l'économie et que, dans leur compréhension de la science économique, le débat entre " primitivistes » et " modernistes " n’avait pas forcément la même importance que dans le cadre des conflits autour de la méthode de l'École historique allemande de l'économie nationale.

Rostovtzeff avait entrepris ses « voyages allemands » à une époque où l'École historique allemande de l'économie nationale dominait encore la science économique, bien que cette position dominante fût déjà attaquée, de l'extérieur, par l'École autrichienne et, de l'intérieur, par de jeunes économistes comme Max Weber et Werner Sombart. Le conflit entre Eduard Meyer et Karl Bücher sur le caractère de l'économie antique ne fut qu'un épisode de la crise de l'École historique. Ces économistes-historiens avaient voulu découvrir les lois de l'économie par un inventaire aussi complet que possible de faits économiques de toutes les époques, et ils avaient échoué. Mais ce ne furent pas des historiens " modernistes " comme Eduard Meyer qui apportèrent la solution à cette crise. Au contraire, la réponse naquit à l'intérieur de l'École historique, au sein de la troisième génération. Il faut mentionner ici d'abord le travail fondamental de Bücher sur "Gewerbe " (industrie) 50, paru dans le Handwörterbuch der Staatswissenschaften. Sombart avait ensuite introduit le concept de "système économique " dans la réflexion des économistes et dans l'histoire économique ; Weber avait montré comment l'économie doit s'appuyer sur une analyse historique et comparative et comment l'historien peut tirer profit des conceptualisations que lui offrent les autres sciences sociales, l'économie ou la sociologie économique en particulier. Weber avait cherché à concilier histoire et théorie économique et à dépasser l'opposition, finalement stérile, entre ces deux voies, dans la fameuse "querelle des méthodes " qui avait fait rage au début du $\mathrm{XXe}$ siècle. Pour lui, théorie (économique) voulait dire rationale Begriffs-, Typen- und Systembildung (construction rationnelle de concepts, de types et de systèmes) 51 et, avec de tels concepts rationnels, l'économiste fournissait des instruments précieux à

50 Cf. maintenant P. SPAHN, "Max Weber et la typologie des modes d'activités industrielles de Karl Bücher", in BRUHNS \& ANDREAU (éd.), Sociologie économique et économie de l'Antiquité, p. 115-132.

51 W. SOMBART \& M. WeBER, "Erklärung”, Archiv für Sozialwissenschaften und Sozialpolitik, 44 (1917), p. 348 (en réponse à E. JAFFÉ, "Das theoretische System der kapitalistischen Wirtschaftsordnung", ibid., p. 1-18). Cf. H. BruHns, "Lectures économiques de Max Weber", La Pensée 314 (1998), p. 39-55. 
l'historien. Rostovtzeff, si proche de Weber sur beaucoup de points, et avec ses connaissances immenses et sa maîtrise de la documentation la plus diverse, aurait pu être la personne idéale pour exploiter ces potentialités au profit de l'histoire ancienne. Est-ce la difficulté du dialogue entre histoire et économie qui a fait que cette rencontre n'a pas eu lieu 52 ?

\author{
Hinnerk BRUHNS \\ Centre de Recherches Historiques \\ CNRS / EHESS \\ 54, Bd Raspail F-75006 Paris \\ bruhns@msh-paris.fr
}

52 Cf. le commentaire de Fernand Braudel, répondant à une question de Jean Andreau et Roland Etienne [" Vous disiez que pour l'Antiquité on ne parvenait pas à faire l'histoire économique. Pourquoi ?»] : "Rostovtseff n'avait pas la formation suffisante. Il fait une histoire économique événementielle. Ce n'est pas là le problème. Le problème, c'est de supposer certaines structures, de montrer que des structures sont en rapport avec les faits connus, et, en partant de cet accord, de reprendre l'ensemble, - quitte à se tromper. " (F. BRAUDEL, "L'Antiquité et l'histoire ancienne", Quaderni di storia 24 [1986], p. 5-21, ici p. 17). 